\title{
Pathological and Immunohistochemical Findings of Natural Highly Pathogenic Avian Influenza Infection in Tufted Ducks during 2010-2011 Outbreaks in Japan
}

\author{
Walied ABDO ${ }^{1,4)}$, Mohie HARIDY ${ }^{1,2)}$, Yuki KATOU ${ }^{1)}$, Minami GOTO ${ }^{1)}$, Toshio MIZOGUCHI ${ }^{3)}$, Yoshihiro SAKODA ${ }^{5)}$, \\ Hiroki SAKAI ${ }^{1)}$ and Tokuma YANAI ${ }^{1) *}$ \\ ${ }^{1)}$ Department of Pathogenetic Veterinary Sciences, United Graduate School of Veterinary Sciences, Gifu University, 1-1 Yanagido, Gifu \\ 501-1193, Japan \\ 2) Department of Pathology and Clinical Pathology, Faculty of Veterinary Medicine, South Valley University, Qena 83523, Egypt \\ 3) Fukushima Wildlife Rehabilitation Center, Adatara-gun, Fukushima 969-1302, Japan \\ 4) Department of Pathology, Faculty of Veterinary Medicine; Kafr El-Sheikh University, Egypt \\ ${ }^{5)}$ Laboratory of Microbiology, Department of Disease Control, Graduate School of Veterinary Medicine, Hokkaido University, Sapporo \\ 060-0818, Japan
}

(Received 17 February 2014/Accepted 12 May 2014/Published online in J-STAGE 30 May 2014)

ABSTRACT. In the winter of 2010-2011, an outbreak of highly pathogenic avian influenza virus (HPAIV) infection occurred in wild and domestic birds in Japan. Tufted ducks were found dead in an urban area of Toyota City, Koriyama, Fukushima Prefecture. Two tufted ducks were examined histopathologically, immunohistochemically and molecularly. Gross findings included marked dark-red clotted blood in the pectoral muscles and multifocal hemorrhages on the serous membranes. Microscopically, non-suppurative meningoencephalitis, multifocal to coalescing pancreatic necrosis and severe pulmonary congestion were observed. HPAIV antigen was detected in the malacic areas, neuronal, glial and ependymal cells, pulmonary capillary endothelial cells and epithelium of pulmonary bronchioles, necrotic pancreatic acini and degenerated cardiac myocytes. The HPAIV isolate was genetically classified into clade 2.3.2.1 group A. The broad distribution of virus antigen in brain and pulmonary tissues associated with HPAIV spontaneous infection in tufted ducks might be useful in understanding its pathogenesis in nature.

KEY WORDS: HPAIV, Japan, outbreak, tufted duck

doi: 10.1292/jvms.14-0084; J. Vet. Med. Sci. 76(9): 1285-1290, 2014

Influenza A viruses (family Orthomyxoviridae) naturally infect a broad range of avian and mammalian species, including humans. Highly pathogenic avian influenza virus (HPAIV) subtype H5N1 is an infectious systemic viral disease that results in high morbidity and mortality in poultry and has been reported in a wide range of wild bird species during the last few years $[13,18]$. In Japan, HPAIV H5N1 has been isolated from several wild birds, including jungle crow, mountain hawk-eagle and whooper swan in 2004, 2007 and 2008, respectively [12, 15, 17]. In autumn 2010, H5N1 was isolated from fecal samples of migrating ducks in the northernmost part of Japan [6]. In the winter of 2010-2011, several outbreaks occurred in wild birds and on domestic farms in Japan, and H5N1 was isolated from 63 wild birds and 24 chicken farms by the end of March 2011 [11]. Forty of the 63 wild birds belong to the order Anseriformes. The dominant deceased birds were tufted ducks, Aythya fuligula (12), from 4 prefectures: Fukushima (5), Tottori (2), Shimane (4) and Yamaguchi (1) [11].

\footnotetext{
*Correspondence to: Yanai, T., Department of Pathogenetic Veterinary Sciences, United Graduate School of Veterinary Sciences, Gifu University, 1-1 Yanagido, Gifu 501-1193, Japan.

e-mail: yanai@gifu-u.ac.jp

(C2014 The Japanese Society of Veterinary Science

This is an open-access article distributed under the terms of the Creative Commons Attribution Non-Commercial No Derivatives (by-nc-nd) License $<$ http://creativecommons.org/licenses/by-nc-nd/3.0/>.
}

The tufted duck breeds across northern Eurasia from Iceland to the Bering Sea. A large proportion (probably more than $60 \%$ ) breeds in Russia and neighboring Fennoscandia. It is mainly migratory, but some populations in Europe are resident or perform only short-distance movements. The birds winter in northwestern and southern Europe, around the Caspian and Black Seas, in northern Africa and across Asia to Japan in the east [4]. The pathology of the spontaneous infection in wild birds is limited to a few species and a small number of birds, because of the difficulty of obtaining fresh carcasses suitable for pathological investigation. Moreover, level of mortality due to HPAI H5N1, although it depends on the wild bird species, is not as high as seen on domestic poultry farms. Here, we describe the gross, histopathological and immunohistochemical characteristics of spontaneous HPAIV infection (H5N1) in 2 tufted ducks and the molecular characterization of the virus isolated from the third duck.

In January 2011, numerous tufted ducks were found dead on a pond in Toyota City in Koriyama, Fukushima Prefecture, in an urban area. Two tufted ducks were examined by means of histopathological, immunohistochemical and molecular investigation, and a third duck was sent for further virus molecular characterization.

The 2 tufted ducks were sent to Gifu University for necropsy and histopathological investigation, and the third duck was sent to Hokkaido University for virus isolation. The ducks were necropsied in an isolated room in the P3 facility at the Faculty of Applied Biological Sciences, Gifu 


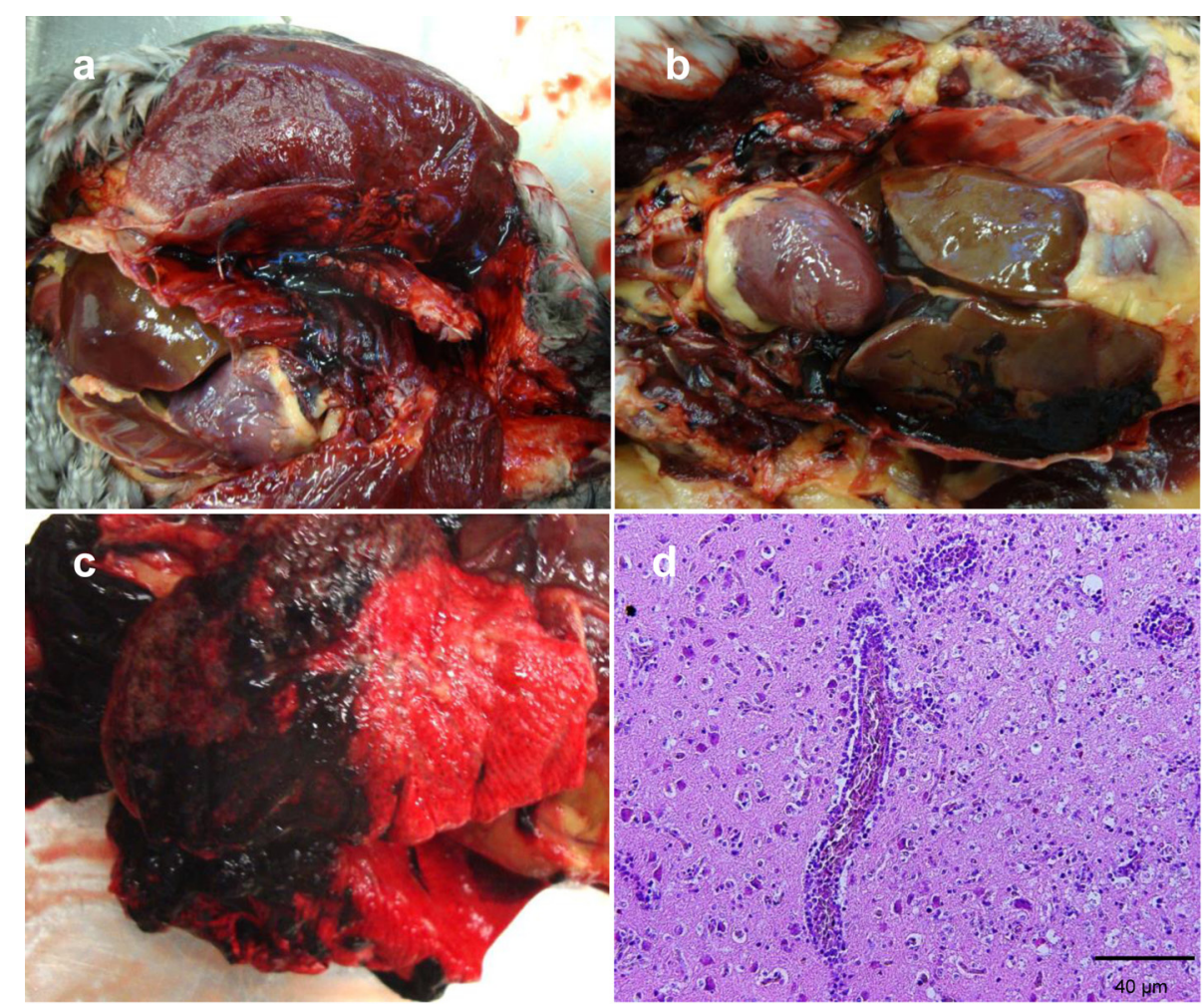

Fig. 1. Gross and histopathological findings of HPAIV in tufted ducks. Lesions were characterized grossly by (a) marked dark-red clotted blood in the pectoral muscles and (b) multifocal hemorrhages on the serosal surface of the liver and pericardium and (c) in the lungs. (d) The histopathological findings in brain of tufted ducks were non-suppurative meningoencephalitis characterized by perivascular mononuclear cell cuffing, multifocal neuronal degeneration and gliosis.

University. Samples from the brain, liver, lungs, pancreas, spleen, kidneys, hearts and adrenals were fixed, trimmed and processed in paraffin using the paraffin embedding technique for histopathological examination. For immunohistochemical investigation, deparaffinized and rehydrated $5-\mu \mathrm{m}$ tissue sections were quenched for $10 \mathrm{~min}$ in aqueous $3 \% \mathrm{H}_{2} \mathrm{O}_{2}$, rinsed in MilliQ water and placed into Tris-buffered saline plus Tween (TBST) buffer for $5 \mathrm{~min}$. The sections were pretreated with proteolytic enzyme (Dako Cytomation, Carpinteria, CA, U.S.A.) for $15 \mathrm{~min}$, rinsed twice with TBST and incubated for $1 \mathrm{hr}$ with a monoclonal antibody specific for influenza A nucleoprotein (Clone 1331, Biodesign, Sasco, ME, U.S.A.) at a dilution of 1:5,000. The sections were washed with TBST and then incubated for $30 \mathrm{~min}$ with the Envision + anti-mouse (horse radish peroxidase-labeled) polymer kit (Dako Cytomation), followed by a TBST rinse. Diaminobenzidine was used as the substrate chromagen, and the slides were counterstained with hematoxylin. Each immunostain includes a positive reference control (intestine from a chicken inoculated by $\mathrm{H} 5 \mathrm{~N} 1$ ), a negative control (brain from a healthy chicken) and a primary-antibody omitted control. For influenza virus diagnosis, viral RNA was extracted from cloacal swab from the 3 tufted ducks using a Qiagen viral RNA minikit according to the manufacturer's instructions. The rRT-PCR was performed using the Qiagen one-step RT-PCR for detection of influenza A matrix gene per manufacturer's instructions. The third duck was sent to laboratory of Microbiology, Department of Disease Control, Graduate School of Veterinary Medicine, Hokkaido University for virus isolation and further molecular characterization.

Grossly, the birds showed normal body mass (weighing $2.520 \& 1.7 \mathrm{~kg}$, both females) with salivation, eye discharge and bilateral bloody discharge. The main gross pathological features were marked dark-reddish clotted blood in the pectoral muscles (Fig. 1a) and multifocal hemorrhages on the serosal surfaces of the pancreas, gizzard, liver (Fig. 1b), pericardium and lungs (Fig. 1c). Splenomegaly, impaction of the proventriculus and gizzard and distension of the intestine with muddy green, white clay-like flakes, were observed. Congestion of the meningeal and cerebral blood vessels and hemorrhages on the surfaces of the parietal and occipital cerebral lobes were also noticed.

Microscopically, the 2 ducks had non-suppurative meningoencephalitis (Fig. 1d) characterized by perivascular lymphocytic cuffing and mild perivascular edema, multifocal neuronal degeneration and vacuolation of the neuropil. Subcortical multifocal areas of liquefactive necrosis associated with gliosis were observed in 1 duck. The perivascular cuffing was composed of mononuclear phagocytic cells 
and lymphocytes. The lungs revealed hemorrhages, severe congestion and mild interstitial mononuclear cell infiltration. Necrotic bronchitis with desquamation of the lining epithelium and fibrino-necrotic airsacculitis was also observed. Other findings included multifocal to coalescing necrosis of the pancreatic acinar epithelium accompanied by an extensive area of severe hemorrhage and surrounded by mononuclear and heterophil cellular infiltration. Vacuolar hepatic degeneration and periportal lymphoplasmacytic infiltrations, hemosidrosis and bile stasis were observed. Also, multifocal hepatic necrosis accompanied by heterophils and lymphocytic infiltration was seen. The heart showed multifocal mild myocarditis with lymphocyte and macrophage infiltration. Myocardial vacuolation was also observed. Severe hemorrhage and congestion of the renal tissue accompanied by mild interstitial infiltrations of mononuclear cells was noticed. One duck had focal necrosis in the adrenal medullar cells with mild lymphocytic infiltration.

Immunohistochemically, the avian influenza antigen was pantropic in the 2 birds. The antigen was detected in the brain, pancreas, liver, spleen, lungs and heart. There was a strong correlation between the demonstration of viral antigen and the microscopic lesions. Strong viral antigen staining was demonstrated throughout the mulifocal liquefactive necrotic areas of the brain (Fig. 2a). The antigen was also randomly distributed in the brain within ependymal cells (Fig. 2b), neurons, glial cells and epithelium of the choroid associated with inflammatory cell infiltrations. In the lungs, the antigen was detected in the capillary endothelial cells of the air vesicles (Fig. 2c) and the necrotic parabronchial epithelium (Fig. 2d). Although severe necro-hemorrhagic pancreatic lesions were observed, rare viral antigens were frequently detected close to the pancreatic acini, close to areas that exhibited necrosis (Fig. 2e). The viral antigen was expressed in the endocardium or necrotic cardiac myocytes (Fig. 2f), but it was frequently detected in the renal tubular epithelium and splenic macrophages. The antigen was detected in the hepatocytes and Kupffer cells related to focal hepatic necrosis.

Avian influenza virus type A was detected by real time RT-PCR in the cloacal swab of the 3 ducks. HPAIV (H5N1) was isolated from the third duck and other 4 ducks of the same species collected from the same mortality event by laboratory of Microbiology, Department of Disease Control, Graduate School of Veterinary Medicine, Hokkaido University [11]. The multiple basic amino acid sequence (RERRRKR/G), which is a marker of HPAI viruses (OIE, 2011), was found at the cleavage site of the deduced amino acid sequence of the hemagglutinin of the isolate. Based on the phylogenetic analysis of hemagglutinin genes, all H5N1 isolates in the 2010-2011 winter season in Japan were classified into genetic clade 2.3.2.1 and sub-grouped into groups $\mathrm{A}, \mathrm{B}$ and $\mathrm{C}$. The isolates from tufted ducks in this study were genetically closely related each other and were classified into clade 2.3.2.1 group A [11].

Since 1996, highly pathogenic H5N1 virus has been identified in domestic geese in China [19], and outbreaks have spread to encompass many countries in Asia, the Middle
East, Europe and Africa [14]. Such widespread dissemination would require wild birds to excrete the virus in the absence of debilitating disease. HPAIV has occasionally been detected in wild birds near affected poultry flocks, but these birds have played a limited role, or no role, in virus dissemination [14]. In contrast, wild birds are suspected of playing a major role as long-distance virus vectors in the 2010-2011 HPAIV outbreaks. Just before these outbreaks, H5N1 HPAIV was isolated from fecal samples of migrating ducks in the northernmost part of Japan in the autumn of 2010 [6]. Moreover, the virus was isolated from healthy mallard ducks captured in South Korea in 2010 [8]. These circumstances support the assumption that migratory water birds from Siberia Lake could play a role in HPAIV dissemination in domestic poultry.

The most consistent gross lesions in the natural infection of tufted ducks with HPAIV were multifocal hemorrhages in the pectoral muscles, the serosal surfaces of the pancreas, liver, pericardium and lungs and hemorrhages and congestion of the meninges. There were also disseminated and multifocal distributions of lesions, suggesting a hematogenous spread of HPAIV in tufted ducks. In the present study, the antigen was detected in the capillary endothelium of the air vesicles of the lungs. Endotheliotropism of HPAI was not detected in experimentally infected tufted ducks [7]. Naturally, there is no evidence that the HPAI antigen causes actual vascular damage, as it has been detected in the vascular endothelium of the nasal mucosa in only 2 out 17 ducks [1]. In contrast, Brown et al. [3] detected virus antigens in the endothelial cells in most of the visceral organs and the brains in experimentally infected black swans. HPAI antigen was detected in endothelial cells and perivascular phagocytes in chickens and other Galliformes, in contrast to the pathogenesis of HPAIV in domestic and wild ducks characterized by lack of virus replication in endothelial cells and absence of associated vascular damage [2, 7]. Even in chickens, although most of HPAI viruses have endothelium tropism and vascular permeability disorders, some HPAI viruses that have a long incubation period, produce viremia with lack of or minimal vascular endothelial replication and tend to replicate extensively in parenchymal cells of visceral organs [9].

The histopathological lesions were multifocal nonsuppurative encephalitis, multifocal necrotizing pancreatitis, vacuolar hepatic degeneration and periportal lymphocytic infiltrations, focal myocarditis and myocardial degeneration, and pulmonary and renal hemorrhages. The virus antigen was systemically detected. This is in agreement with natural H5N1 infection in tufted ducks and other wild bird species $[1,5,15,16]$. The brain and pancreas lesions were similar to those described in other natural and experimental infections in wild birds. However, in the present study, the non-suppurative meningoencephalitis with multifocal liquefactive necrosis was consistently observed in the 2 ducks with associated strong deep IHC-positive staining in the subcortical multifocal liquefactive necrosis, degenerated neurons and ependymal and glial cells. The high level of antigen detected in the brain suggested the high neurotropism of the virus 


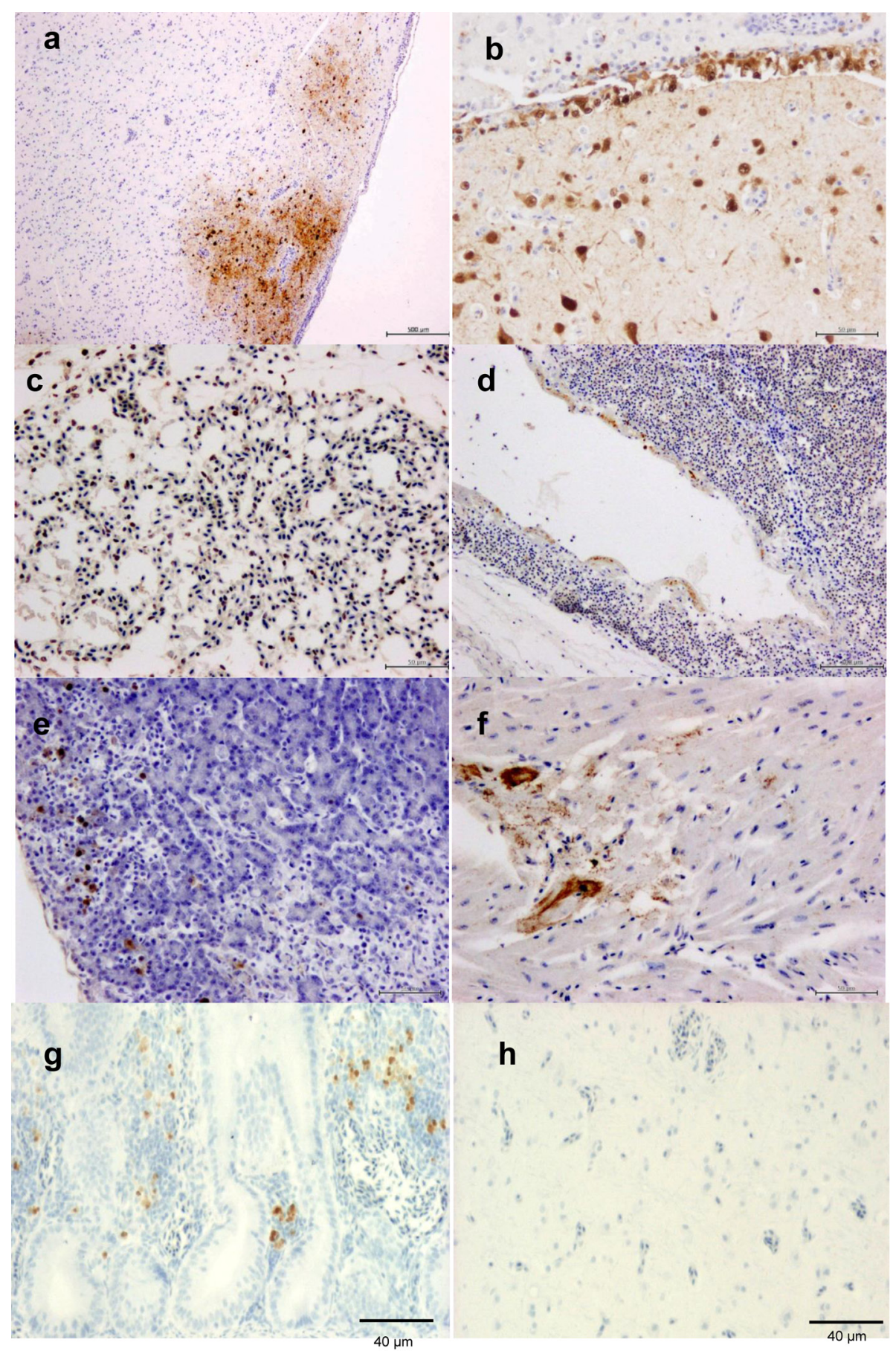

Fig. 2. Immunohistochemical detection of avian influenza antigen in tufted ducks in (a) subcortical multifocal liquefactive necrosis and (b) ependymal, degenerated neurons and glial cells of the brain, (c) the pulmonary endothelium of the alveolar capillary, (d) epithelial cells of the parabronchus and atrium, (e) pancreatic acini and (f) focal degenerated cardiac myocytes as well as in (g) lymphocytic infiltration in lamina propria of the intestine in positive control chicken, but not in (h) brain of negative control chicken (ABC immunoperoxidase technique, counterstained with Mayer's hematoxylin).

that might be responsible for the ultimate death of the birds $[3,7,10,16]$. In contrast, although the pancreas was severely necrotic and hemorrhagic, the immunostaining of the virus antigen was weak and in acini were devoid of degeneration 
surrounding necrotic tissue that was IHC-negative. This suggested that the virus could be preserved in tissues for a short period [1], although this could be contrasted with the positivity of the subcortical liquefactive necrosis in the brain.

In the lungs, hemorrhages, severe congestion and mild interstitial mononuclear cell infiltration accompanied by necrotic bronchitis and fibrino-necrotic airsacculitis were also observed. The virus antigen was detectable in the capillary endothelium, as has previously been recorded in naturally infected tufted ducks [1] and mute and whooper swans [16]. On the other hand, the antigen was detectable in the epithelium of the parabronchus and atrium, unlike earlier records of natural infection in tufted ducks and mute and whooper swans $[1,16]$. In intratracheally and intraesophageally experimentally inoculated tufted ducks, the viral antigen was found in the epithelium of bronchi, parabronchi, atria and air capillaries [7]. Virus detection in pulmonary tissue is dosage- and route-dependent and can vary in natural infection from 1 bird to another. The virus antigen was detected in necrotic cardiac myocytes and frequently in the renal tubular epithelium and splenic reticuloendothelial cells. HPAI viral replication in cardiac myocytes has been detected in both naturally [15, 16] and experimentally [7] infected birds. The hepatic focal degeneration with focal hepatocyte immunopositivity to HPAIV antigen in the 2 cases presented here highlights the pantropism of the virus.

The severity of hemorrhagic and necrotic lesions in the pectoral muscles, lungs, pancreas and brain in the tufted ducks in the present case, as well as the pantropic distribution of the HPAIV antigen in various organs, besides those found dead, suggests that tufted ducks might be more vulnerable to infection than other wild birds, as was previously presumed in experimental infection [7]. The detection of AI antigen in endothelium of air capillaries and epithelium of parabronchi is a new finding that might be helpful to understand the pathogenesis of HPAI in nature.

ACKNOWLEDGMENT. We thank Ms. C. Swift for proofreading the manuscript.

\section{REFERENCES}

1. Bröjer, C., Agren, E. O., Uhlhorn, H., Bernodt, K., Morner, T., Jansson, D. S., Mattsson, R., Zohari, S., Thoren, P., Berg, M. and Gavier-Widen, D. 2009. Pathology of natural highly pathogenic avian influenza H5N1 infection in wild tufted ducks (Aythya fuligula). J. Vet. Diagn. Invest. 21: 579-587. [Medline] [CrossRef]

2. Brown, J. D., Stallknecht, D. E., Beck, J. R., Suarez, D. L. and Swayne, D. E. 2006. Susceptibility of North American ducks and gulls to $\mathrm{H} 5 \mathrm{~N} 1$ highly pathogenic avian influenza viruses. Emerg. Infect. Dis. 12: 1663-1670. [Medline] [CrossRef]

3. Brown, J. D., Stallknecht, D. E. and Swayne, D. E. 2008. Experimental infection of swans and geese with highly pathogenic avian influenza virus (H5N1) of Asian lineage. Emerg. Infect. Dis. 14: 136-142. [Medline] [CrossRef]

4. Delany, S., Veen, J. and Clark, J. A. 2006. Urgent preliminary assessment of ornithological data relevant to the spread of Avian Influenza in Europe. Report to the European Commission. Study contract: 07010401/2005/425926/MAR/B4. 2006; Available from: http://ec.europa.eu/environment/nature/nature_conservation/focus wild birds/avian influenza/index en.htm.

5. Ellis, T. M., Bousfield, R. B., Bissett, L. A., Dyrting, K. C., Luk, G. S., Tsim, S. T., Sturm-Ramirez, K., Webster, R. G., Guan, Y. and Malik Peiris, J. S. 2004. Investigation of outbreaks of highly pathogenic $\mathrm{H} 5 \mathrm{~N} 1$ avian influenza in waterfowl and wild birds in Hong Kong in late 2002. Avian Pathol. 33: 492-505. [Medline] [CrossRef]

6. Kajihara, M., Matsuno, K., Simulundu, E., Muramatsu, M., Noyori, O., Manzoor, R., Nakayama, E., Igarashi, M., Tomabechi, D., Yoshida, R., Okamatsu, M., Sakoda, Y., Ito, K., Kida, H. and Takada, A. 2011. An H5N1 highly pathogenic avian influenza virus that invaded Japan through waterfowl migration. Jpn. J. Vet. Res. 59: 89-100. [Medline]

7. Keawcharoen, J., van Riel, D., van Amerongen, G., Bestebroer, T., Beyer, W. E., van Lavieren, R., Osterhaus, A. D., Fouchier, R. A. and Kuiken, T. 2008. Wild ducks as long-distance vectors of highly pathogenic avian influenza virus (H5N1). Emerg. Infect. Dis. 14: 600-607. [Medline] [CrossRef]

8. Kim, H. R., Kim, B. S., Bae, Y. C., Moon, O. K., Oem, J. K., Kang, H. M., Choi, J. G., Lee, O. S. and Lee, Y. J. 2011. H5N1 subtype highly pathogenic avian influenza virus isolated from healthy mallard captured in South Korea. Vet. Microbiol. 151: 386-389. [Medline] [CrossRef]

9. Pantin-Jackwood, M. J. and Swayne, D. E. 2009. Pathogenesis and pathobiology of avian influenza virus infection in birds. Rev. Sci. Tech. 28: 113-136. [Medline]

10. Pasick, J., Berhane, Y., Embury-Hyatt, C., Copps, J., Kehler, H., Handel, K., Babiuk, S., Hooper-McGrevy, K., Li, Y., Mai Le, Q. and Lien Phuong, S. 2007. Susceptibility of Canada Geese (Branta canadensis) to highly pathogenic avian influenza virus (H5N1). Emerg. Infect. Dis. 13: 1821-1827. [Medline] [CrossRef]

11. Sakoda, Y., Ito, H., Uchida, Y., Okamatsu, M., Yamamoto, N., Soda, K., Nomura, N., Kuribayashi, S., Shichinohe, S., Sunden, Y., Umemura, T., Usui, T., Ozaki, H., Yamaguchi, T., Murase, T., Ito, T., Saito, T., Takada, A. and Kida, H. 2012. Reintroduction of $\mathrm{H} 5 \mathrm{~N} 1$ highly pathogenic avian influenza virus by migratory water birds, causing poultry outbreaks in the 2010-2011 winter season in Japan. J. Gen. Virol. 93: 541-550. [Medline] [CrossRef]

12. Shivakoti, S., Ito, H., Otsuki, K. and Ito, T. 2010. Characterization of $\mathrm{H} 5 \mathrm{~N} 1$ highly pathogenic avian influenza virus isolated from a mountain hawk eagle in Japan. J. Vet. Med. Sci. 72: 459-463. [Medline] [CrossRef]

13. Stallknecht, D. E., Nagy, E., Hunter, D. B. and Slemmons, R. D. 2007. Avian influenza. pp. 108-130. In: Infectious Diseases of Wild Birds (Thomas N. J., Hunter, H. D. and Atkinson C. T. eds.), Blackwell Publishing, Ames.

14. Swayne, D. E. and Halvorson, D. A. 2008. Influenza. pp. 153-184. In: Diseases of Poultry, 12th ed. (Saif, Y.M., Glisson, J. R., Fadly, A. M., McDougald, L. R. and Nolan, L. K. eds.), Blackwell Publishing Professional, Ames.

15. Tanimura, N., Tsukamoto, K., Okamatsu, M., Mase, M., Imada, T., Nakamura, K., Kubo, M., Yamaguchi, S., Irishio, W., Hayashi, M., Nakai, T., Yamauchi, A., Nishimura, M. and Imai, K. 2006. Pathology of fatal highly pathogenic H5N1 avian influenza virus infection in large-billed crows (Corvus macrorhynchos) during the 2004 outbreak in Japan. Vet. Pathol. 43: 500-509. [Medline] [CrossRef]

16. Teifke, J. P., Klopfleisch, R., Globig, A., Starick, E., Hoffmann, B., Wolf, P. U., Beer, M., Mettenleiter, T. C. and Harder, T. C. 2007. Pathology of natural infections by H5N1 highly patho- 
genic avian influenza virus in mute (Cygnus olor) and whooper (Cygnus cygnus) swans. Vet. Pathol. 44: 137-143. [Medline] [CrossRef]

17. Uchida, Y., Mase, M., Yoneda, K., Kimura, A., Obara, T., Kumagai, S., Saito, T., Yamamoto, Y., Nakamura, K., Tsukamoto, K. and Yamaguchi, S. 2008. Highly pathogenic avian influenza virus (H5N1) isolated from whooper swans, Japan. Emerg. Infect. Dis. 14: 1427-1429. [Medline] [CrossRef]
18. Wright, P. F., Neumann, G. and Kawaoka, Y. 2007. Orthomyxoviruses. In: Fields Virology (Knipe, D.M. and Howley, P.M. eds.), Lippincott Williams \& Wilkins, Philadelphia.

19. Xu, X., Subbarao, Cox, N. J. and Guo, Y. 1999. Genetic characterization of the pathogenic influenza A/Goose/Guangdong/1/96 (H5N1) virus: similarity of its hemagglutinin gene to those of H5N1 viruses from the 1997 outbreaks in Hong Kong. Virology 261: 15-19. [Medline] [CrossRef] 